\title{
INFINITE CYCLIC NORMAL SUBGROUPS \\ OF FUNDAMENTAL GROUPS OF NONCOMPACT 3-MANIFOLDS
}

\author{
BOBBY NEAL WINTERS
}

(Communicated by James West)

\begin{abstract}
It is shown that an end-irreducible 3-manifold each of whose boundary components is compact and whose fundamental group contains an infinite cyclic normal subgroup is Seifert fibered.
\end{abstract}

In this paper, it is shown that if $W$ is a noncompact, orientable, irreducible, end-irreducible 3-manifold such that each component of $\partial W$ is compact and $\pi_{1}(W)$ contains an infinite cyclic normal subgroup, then $W$ is Seifert fibered.

This was inspired by the work of Dave Gabai [G] and Andrew Casson [CJ], who showed independantly that if $M$ is a compact, orientable, irreducible 3manifold such that $\pi_{1}(M, *)$ contains an infinite cyclic normal subgroup, then $M$ is a Seifert fibered space. This paper, however, will not need the full strength of this result. Instead Corollary II.6.4 of [JS], in which $M$ is assumed to be sufficiently large, is used.

In this paper, $W$ will always be a noncompact, orientable, irreducible 3manifold such that $\pi_{1}(W, *)$ contains an infinite cyclic normal subgroup $C$, where $* \in W$ is fixed.

If for every compact $K \subset W$ and every noncompact component $U$ of $\operatorname{cl}(W-K)$ there is a loop $\lambda_{U}$ in $U$ that is freely homotopic in $W$ to a generator of $C$, then we say that $C$ is end-peripheral. If for every compact $K \subset W$ there is a compact 3-manifold $M_{K} \subset W$ with $K \subset M_{K}-\operatorname{Fr}\left(M_{K}\right)$ and $\operatorname{Fr}\left(M_{K}\right)$ incompressible in $W$, we say that $W$ is end-irreducible.

In order to prove the result stated in the first paragraph, it is proven that $W$ is Seifert fibered whenever $C$ is end-peripheral and $W$ is end-irreducible. Then it is shown that $C$ is end-peripheral whenever $W$ is end-irreducible and each component of $\partial W$ is compact.

The assumption of $C$ being end-peripheral is not unnecessarily strong. In fact it is not difficult to see that if $\Sigma$ is a noncompact Seifert fibered space and $\Gamma$ is the infinite cyclic normal subgroup of $\pi_{1}(\Sigma, *)$ generated by a regular fiber, then $\Gamma$ is end-peripheral.

Similarly, the assumptions of irreducibility and end-irreducibility are reasonable. By Lemma 2.2 of [W], it follows that $\mathbf{R}^{2} \times S^{1}$ is the only noncompact Seifert fibered space that is not end-irreducible. It is shown in Lemma 2.1 of

Received by the editors February 13, 1992.

1991 Mathematics Subject Classification. Primary 57N10.

(C) 1994 American Mathematical Society 
[W] that every noncompact Seifert fibered space is irreducible.

There do exist homotopy open solid tori with end-peripheral fundamental groups that are not homeomorphic to $\mathbf{R}^{2} \times S^{1}$. One can be constructed as follows. Let $H$ be an open manifold that is a union of solid tori $\left\{V_{n} \mid n \geq 0\right\}$ such that, for $n \geq 1, V_{n} \subset V_{n+1}-\partial V_{n+1}$ and the core of $V_{n}$ is homotopic in $V_{n+1}$ to the core of $V_{n+1}$. It is easy to show that $\pi_{1}(H, *)=\mathbf{Z}$ and that $\pi_{1}(H, *)$ is end-peripheral. However, it is possible to construct $H$ so that $H$ is not an open solid torus. (See $M_{2}$ of [ST], for example.) Such an $H$ is not Seifert fibered because $H$ is not end-irreducible. However, it is possible that another hypothesis could replace end-irreducibility. For instance, Mess has shown in [M] that if $\hat{M}$ is a noncompact 3-manifold such that $\pi_{1}(\hat{M}, *)$ is an abelian group of rank 1 which is a regular cover of a closed, irreducible 3-manifold, then $\hat{M}$ is an open solid torus and, therefore, Seifert fibered. In Lemma 3 of Theorem 1 of his proof, Mess actually shows that $\pi_{1}(\hat{M}, *)$ is end-peripheral, though he does not use this terminology.

There do exist examples of end-irreducible 3-manifolds whose fundamental groups contain non-end-peripheral infinite cyclic normal subgroups. Let $F$ be a compact, connected 2-manifold with $\partial F \neq \varnothing$ that is not an annulus or a disk. Let $J$ be a component of $\partial F$. Let $M=F \times S^{1}$ and $T=J \times S^{1}$. Let $x \in J$, $l=x \times S^{1}$, and $m=J \times 1$. Let $L$ be a simple closed curve in $T$ which is equal to $l^{2} m^{3}$ in $\pi_{1}(T, *)$. Let $M^{\prime}=M-L$. Then $M^{\prime}$ is irreducible. Let $K$ be a compact subset of $M^{\prime}$. Let $V$ be a regular neighborhood of $L$ in $M-K$. Then $V$ is a solid torus whose core is homotopic in $V$ to $L$. Let $M_{K}=\operatorname{cl}(M-V)$. Then $\operatorname{Fr}\left(M_{K}\right)$ is incompressible in $M^{\prime}$, so $M^{\prime}$ is endirreducible. Note that $\pi_{1}\left(M^{\prime}, *_{1}\right)$ contains an infinite cyclic normal subgroup that is generated by $*_{1} \times S^{1}$, where $*_{1} \in F-\partial F$. However, $M^{\prime}$ is not Seifert fibered because $\left\langle *_{1} \times S^{1}\right\rangle$ is not end-peripheral. This is because any loop in $V \cap M^{\prime}$ is homotopic in $V$ to a power of $l^{2} \mathrm{~m}^{3}$ and, therefore, cannot be freely homotopic in $M^{\prime}$ to $*_{1} \times S^{1}$ or its inverse.

\section{INFINITE CYCLIC NORMAL SUBGROUPS}

Let $p: \tilde{W} \rightarrow W$ be the cover of $W$ such that $p_{*}\left(\pi_{1}(\tilde{W}, \tilde{*})\right)=C$, where $\tilde{*} \in \tilde{W}$ with $p(\tilde{*})=*$. By [S] there is a compact 3-manifold $L \subset \tilde{W}$ such that $j_{*}: \pi_{1}(L, \tilde{*}) \rightarrow \pi_{1}(\tilde{W}, \tilde{*})$ is an isomorphism where $j: L \rightarrow \tilde{W}$ is the inclusion map. We shall say that a compact 3-submanifold $M$ of $W$ is $C$-aware if $p(L) \subset M$.

Lemma 1. Let $M \subset W$ be a $C$-aware compact 3-manifold, and let $i: M \rightarrow W$ be the inclusion map.

(1) $C$ is a subgroup of $i_{*} \pi_{1}(M, *)$.

(2) If $i_{*}$ is injective and no component of $\partial M$ is a sphere, then $M$ has a Seifert fibering in which each fiber is freely homotopic in $M$ to a generator of $C$.

Proof. To prove (1), let $q: L \rightarrow M$ be defined by $q(x)=p(x)$ for every $x \in L$. Then $i q=p j$. So $i_{*} q_{*}=p_{*} j_{*}$. Therefore, $C=i_{*} q_{*} \pi_{1}(L, \tilde{*})$.

To prove (2), suppose that $i_{*}$ is injective. Then $\pi_{1}(M, *)$ contains an infinite cyclic normal subgroup. It is not difficult to show that $M$ is Haken. Therefore, by Corollary II.6.4 of [JS], it follows that $M$ is Seifert fibered in the way described in (2). 
For the rest of the section, suppose that every compact $K \subset W$ is contained in a compact 3-submanifold $M_{K}$ of $W$ such that the inclusion induced map $\pi_{1}\left(M_{K}, *\right) \rightarrow \pi_{1}(W, *)$ is injective.

Proposition 2. Every compact component of $\partial W$ is a torus.

Proof. Let $F$ be a compact component of $\partial W$. Let $M_{F}$ be a compact 3submanifold of $W$ such that $F \subset M_{F}-\operatorname{Fr}\left(M_{F}\right)$ and the inclusion induced map $\pi_{1}\left(M_{F}, *\right) \rightarrow \pi_{1}(W, *)$ is injective. We may assume that $M_{F}$ is $C$-aware and that no component of $\mathrm{cl}\left(W-M_{F}\right)$ is compact. By Lemma 1, it follows that $M_{F}$ is Seifert fibered. Since $F$ is a component of $\partial M_{F}$, it follows that $F$ is a torus.

Proposition 3. If $W$ is not end-irreducible and $\partial W$ is compact, then for every compact $K \subset W$ there is a solid torus $V$ such that $K \subset V-\operatorname{Fr}(V)$. In particular, $\partial W=\varnothing$ and $\pi_{1}(W, *)$ is a subgroup of $(\mathbf{Q},+)$.

Proof. Let $K \subset W$ be compact. We may assume that $\partial W \subset K$. Let $V$ be a compact, $C$-aware 3-manifold in $W$ with $K \subset V-\operatorname{Fr}(V)$ such that no component of $\operatorname{cl}(W-V)$ is compact and the inclusion induced map $\pi_{1}(V, *) \rightarrow$ $\pi_{1}(W, *)$ is injective. It follows easily that $V$ must be Haken. By Lemma 1 , it follows that $V$ is Seifert fibered.

We claim that $V$ is a solid torus. Since $W$ is not end-irreducible, we may choose $V$ so that $\operatorname{Fr}(V)$ is compressible in $W$. Note that each component of $\operatorname{Fr}(V)$ is a component of $\partial V$ because $\partial W \subset V-\operatorname{Fr}(V)$. Consequently each component of $\operatorname{Fr}(V)$ is a torus. Suppose that $D$ is a compressing disk for $\operatorname{Fr}(V)$ in $W$.

We claim that $D \subset V$. Suppose that $D \subset \operatorname{cl}(W-V)$. Let $T$ be the component of $\operatorname{Fr}(V)$ that contains $\partial D$. Then $T$ is a torus. By compressing $T$ along $D$, we can obtain a 2-sphere $S$ which must bound a 3-cell $B \subset W$. Since no component of $\mathrm{cl}(W-V)$ is compact, it follows that $V \subset B$. Since $V$ is $C$-aware, this is a contradiction.

Since $D \subset V$, it follows $V$ is a solid torus. It is an exercise to show that $\pi_{1}(W, *)$ can be embedded in $(\mathbf{Q},+)$.

\section{END-PERIPHERAL SUBGROUPS}

In this section, we shall suppose that $C$ is end-peripheral and $W$ is endirreducible.

Let $M$ be a $C$-aware compact, connected 3-manifold in $W$ such that $\operatorname{Fr}(M)$ is incompressible in $W$ and no component of $\operatorname{cl}(W-M)$ is compact. Let $N$ be a compact, connected 3-manifold in $W$ with $M \subset N-\operatorname{Fr}(N)$ such that $\operatorname{Fr}(N)$ is incompressible in $W$, no component of $\operatorname{cl}(W-N)$ is compact, and each component of $\operatorname{cl}(W-M)$ meets only one component of $\operatorname{cl}(N-M)$. Let $Q$ be a component of $\operatorname{cl}(N-M)$, and let $M_{Q}=M \cup Q$.

Lemma 4. For some $*_{1} \in Q \cap \operatorname{Fr}(M), \pi_{1}\left(Q, *_{1}\right)$ contains an infinite cyclic subgroup $G$ that is normal in $\pi_{1}\left(W, *_{1}\right)$ (and so in $\pi_{1}\left(Q, *_{1}\right)$ ) whose generator is freely homotopic in $W$ to a generator of $C$.

Proof. Let $U$ be the component of $\operatorname{cl}(W-M)$ that contains $Q$. There is a loop $\lambda^{\prime}: S^{1} \rightarrow W$ with $\lambda^{\prime}\left(S^{1}\right) \subset U$ that is freely homotopic in $W$ to a 
generator of $C$. By the usual arguments involving the incompressibility of $\operatorname{Fr}(Q)$ in $W$, it follows that $\lambda^{\prime}$ is freely homotopic in $U$ to a loop $\lambda: S^{1} \rightarrow U$ with $\lambda\left(S^{1}\right)$ contained in $Q \cap \operatorname{Fr}(M)$. Let $*_{1}=\lambda(1)$, and let $G$ be the subgroup of $\pi_{1}\left(Q, *_{1}\right)$ that is generated by $\lambda$.

We claim that $G$ is an infinite cyclic normal subgroup of $\pi_{1}\left(Q, *_{1}\right)$. Since $\operatorname{Fr}(Q)$ is incompressible in $W$, we may consider $\pi_{1}\left(Q, *_{1}\right)$ to be a subgroup of $\pi_{1}\left(W, *_{1}\right)$. Note that $\lambda$ is freely homotopic in $W$ to a generator of $C$. Let $\Lambda: S^{1} \times I \rightarrow W$ be a map such that $\Lambda(z, 0)=\lambda(z)$ and $\Lambda \mid S^{1} \times 1$ generates $C$. Let $\alpha: I \rightarrow W$ be the path from $*_{1}$ to $*$ defined by $\alpha(t)=\Lambda(1, t)$. Let $\phi_{\alpha}: \pi_{1}\left(W, *_{1}\right) \rightarrow \pi_{1}(W, *)$ be the change of base-point isomorphism along $\alpha$. Then $\phi_{\alpha}(\lambda)$ generates $C$. Therefore, $\phi_{\alpha}(G)=C$. So $\phi_{\alpha}(G)$ is normal in $\phi_{\alpha}\left(\pi_{1}\left(W, *_{1}\right)\right)$. Therefore, $G$ is normal in $\pi_{1}\left(W, *_{1}\right)$.

\section{Lemma 5. $W$ is Seifert fibered.}

Proof. By Lemma 1, $M$ is Seifert fibered with fiber freely homotopic in $M$ to a generator of $C$.

By Lemma $4, \pi_{1}\left(Q, *_{1}\right)$ contains an infinite cyclic normal subgroup $G$ whose generator $g$ is freely homotopic in $W$ to a generator $c$ of $C$. In particular, it follows from Corollary II.6.4 of [JS] that $Q$ is Seifert fibered with fiber freely homotopic in $Q$ to $g$.

Let $\Lambda: S^{1} \times I \rightarrow W$ be a map such that $g=\Lambda \mid S^{1} \times 0$ and $c=\Lambda \mid S^{1} \times 1$. Let $\alpha: I \rightarrow W$ be defined by $\alpha(t)=\Lambda(1, t)$. Then $\alpha$ is a path in $W$ from $*_{1}$ to $*$. Let $\phi_{\alpha}: \pi_{1}\left(W, *_{1}\right) \rightarrow \pi_{1}(W, *)$ be the change of base-point isomorphism along $\alpha$. Let $\beta$ be a path in $M$ from $*_{1}$ to $*$, and let $\phi_{\beta}: \pi_{1}\left(W, *_{1}\right) \rightarrow \pi_{1}(W, *)$ be the change of base-point isomorphism along $\beta$.

Given a path $f: I \rightarrow W$, let $\hat{f}: I \rightarrow W$ be defined by $\hat{f}(t)=f(1-t)$. Therefore, $\beta \hat{\alpha}$ and $\alpha \hat{\beta}$ are loops in $W$ based at $*_{1}$ and $(\beta \hat{\alpha})(\alpha \hat{\beta})$ is homotopic with $*_{1}$ fixed to $*_{1}$. Since $G$ is normal in $\pi_{1}\left(W, *_{1}\right)$, it follows that $\beta \hat{\alpha} g \alpha \hat{\beta}$ is homotopic with $*_{1}$ fixed to $g^{\epsilon}$, where $|\epsilon|=1$. Hence $\phi_{\beta}\left(g^{\epsilon}\right)=$ $\hat{\alpha} g \alpha$, and $\hat{\alpha} g \alpha$ is homotopic with $*$ fixed to $c$. Therefore, $\phi_{\beta}\left(g^{\epsilon}\right)=c$. Since $\phi_{\beta}\left(g^{\epsilon}\right)$ and $c$ are loops in $M$ and the inclusion induced map $\pi_{1}(M, *) \rightarrow$ $\pi_{1}(W, *)$ is injective, $\phi_{\beta}\left(g^{\epsilon}\right)$ and $c$ are homotopic in $M$.

It is now easy to see that we may isotop the fibering of $Q$ by an isotopy fixed off a neighborhood of $\partial Q \cap M$ in such a way that each fiber of $Q \cap \partial M$ is a fiber of $M \cap \partial Q$. That is, we may extend the fibering of $M$ to $M_{Q}$. Hence we may extend the fibering of $M$ to $N$. ing.

Since $W$ is end-irreducible, it follows that $W$ can be given a Seifert fiber-

\section{COMPACT BOUNDARY COMPONENTS}

For the rest of the paper, it will be assumed that $W$ is end-irreducible and that each component of $\partial W$ is compact.

Lemma 6. $C$ is end-peripheral.

Proof. Suppose that $K \subset W$ is compact. Let $U$ be a noncompact component of $\operatorname{cl}(W-K)$. Let $M$ be a compact, connected, $C$-aware 3-manifold such that $\operatorname{Fr}(M)$ is incompressible in $W$ and such that $M-\operatorname{Fr}(M)$ contains $K$ and every 
component of $\partial W$ that meets $K$. Let $F$ be the union of components of $\partial M$ that do not meet $K$. Let $F^{\prime}=F \cap U$. Since $M-\operatorname{Fr}(M)$ contains $K$ and all of the components of $\partial W$ that meet $K$, it follows that $F^{\prime}$ is a nonempty union of components of $\partial M$.

By Lemma 1 , it follows that $M$ is fibered with fibers that are freely homotopic to a generator of $C$. Let $\lambda$ be a fiber of $M$ that is contained in $F^{\prime}$. This ends the proof.

Theorem 7. $W$ is Seifert fibered.

Proof. This follows by Lemmas 5 and 6.

\section{REFERENCES}

[G] David Gabai, Convergence groups are Fuchsian groups, preprint.

[CJ] Andrew Casson and Douglas Jungreis, Convergence groups and Seifert fibered 3-manifolds, preprint.

[JS] William H. Jaco and Peter B. Shalen, Seifert fibered spaces in 3-manifolds, Mem. Amer. Math. Soc., vol. 21, no. 220, Amer. Math. Soc., Providence, RI, 1979.

[M] G. Mess, Centers of 3-manifold groups and groups which are coarse quasiisometric to planes, preprint.

[S] G. P. Scott, Compact submanifolds of 3-manifolds, J. London Math. Soc. (2) 7 (1973), 246-250.

[STu] Peter Scott and Thomas Tucker, Some examples of exotic non-compact 3-manifolds, Quart. J. Math. Oxford 40 (1989), 481-499.

[W] Bobby Neal Winters, Planes in 3-manifolds of finite genus at infinity (in preparation).

Department of Mathematics, Pittsburg State University, Pittsburg, Kansas 66762

E-mail address: vinters@ukavm.bitnet 